\title{
Magnetic field evolution in magnetars and its implication to accreting system
}

\author{
Yasufumi Kojima \\ Department of Physics, Hiroshima University, Higashi-Hiroshima, 739-8526, Japan \\ email: kojima@theo.phys.sci.hiroshima-u.ac.jp
}

\begin{abstract}
The evolution of magnetic field is numerically studied for an isolated magnetar, assuming vacuum exterior. Nonlinear coupling between poloidal and toroidal components of the magnetic field can be seen in the initial Hall-drift timescale. Consequently, the polar field at the surface is highly distorted during the phase. This result is suggestive. Fixed dipole magnetic field has been used so far in the theoretical study of the interaction between magnetosphere and accreting matter. In the accretion to magnetar, time-dependent polar magnetic field should be taken into account.
\end{abstract}

Keywords. magnetic fields, stars: neutron

\section{Overview}

Evolution of magnetic field is important, and may account for the variety of field strength among neutron stars. One important factor is an accretion into the central star in a binary system. In most works (e.g, Ghosh \& Lamb (1979)), the field configuration is assumed to be fixed, and the strength decays due to Ohmic dissipation. This picture may not be true for a magnetar. Recently, we have calculated the evolution of the magnetic fields confined in the crust in an isolated system. In strong magnetic field regime $(B>$ $10^{14} \mathrm{G}$ ), the Hall drift is very important. The equation becomes non-linear for the field strength, and the dynamics should inevitably be complicated.

Our numerical results are given in Figure 1. (Kojima \& Kisaka (2012), see also Hollerbach \& Rüdiger (2004), Pons \& Geppert (2007).) The initial configuration is given by the $l=1$ component for poloidal and toroidal fields. Oscillatory behavior is clearly evident in the magnetic flux functions $G$ and $S$, by which the magnetic field is given as $\vec{B}=\left(\vec{\nabla} G \times \vec{e}_{\phi}\right) / R+(S / R) \vec{e}_{\phi}$. This kind of oscillatory behavior is evident only when the torodial field dominates. Typical timescale of the variation is the Hall drift one $\sim 10^{5-6}\left(B_{0} / 10^{13} \mathrm{G}\right)^{-1}$ years, which is much smaller than that of Ohmic decay $\sim 10^{8-9}$ years. Contrarily, if the dipole dominates initially, the configuration is stable until its decay on a timescale $\sim 10^{8-9}$ years. There is a great ambiguity in the field-strength and configuration of the initial magnetic fields, so that the theoretical model at present can not predict unique evolutionary track, but gives a hint to the observation.

\section{Implications}

All magnetars observed so far are isolated ones. At moment, there is no theory to explain the magnetic field-strength at birth, although that of magnetars is thought to be amplified somewhat. Some star is possibly born in a binary system. We briefly discuss such a hypothetical situation, i.e, a magnetar hidden in accreting system. See also Li \& van den Heuvel (1999) for such a candidate in very slowly rotating pulsar. Magnetic coupling between the disk and star is very crucial, and may be quite different in nature 

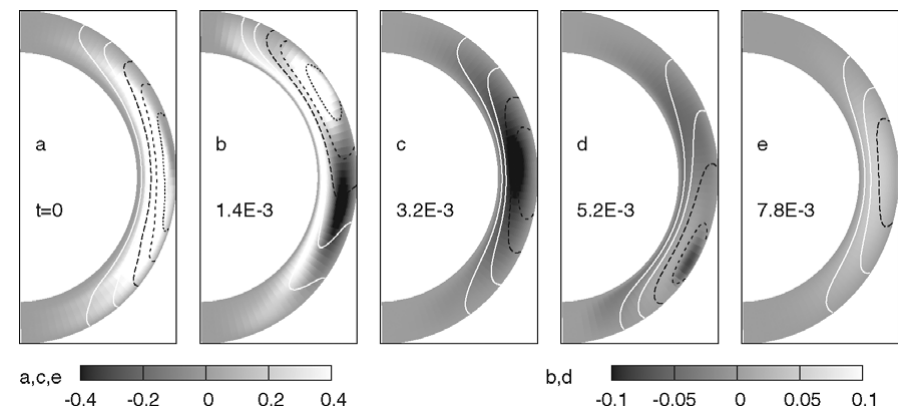

Figure 1. Snapshots of the evolving fields, $G$ and $S$ at representative times. The gray-scale contour represents the function $S$ of the toroidal field, and lines denote the contour of the magnetic flux function $G$ of the poloidal field. Contour lines outwardly represent the level of $G$ for $0.02 \times n \times\left(B_{0} r_{s}^{3}\right), n=1,2, \cdots$. Gray-scale contour represents $S$ normalized by $B_{0} r_{s}$. Note that different scales are used, since $S$ becomes very small at the bounces in the second and fourth panels. Those panels use the gray scale on the left; the others, the scale on the right. Color scale version is available in Kojima \& Kisaka (2012).
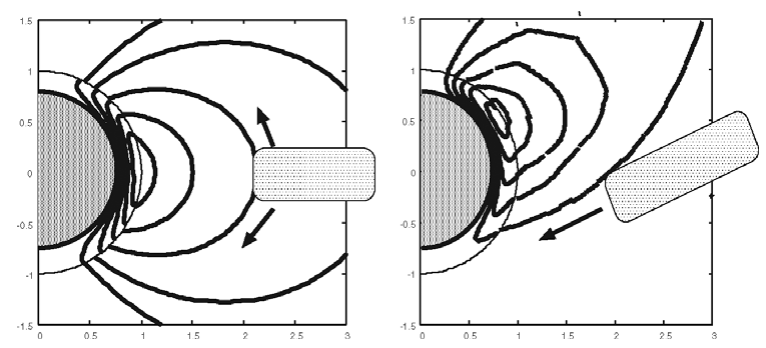

Figure 2. Schematic accretion flow around symmetric dipole magnetic field(left) and distorted one(right). The configuration of right panel corresponds to the second panel in Figure 1.

from that for ordinary X-ray pulsar, since the magnetic field configuration is variable on a Hall drift timescale $\sim 10^{5}$ years. See Figure 2. Accretion onto a star with non-dipole is studied by numerical MHD simulations (e.g, Long, Romanova \& Lovelace (2008)). The simulation range is within several times rotational period. The long-term effect remains as one of interesting theoretical issues.

\section{References}

Ghosh, P. \& Lamb, F. K. 1979, ApJ, 234, 296

Hollerbach, R. \& Rüdiger, G. 2004, MNRAS, 347, 1273

Kojima, Y. \& Kisaka, S. 2012, MNRAS, 421, 2722

Li, X. D. \& van den Heuvel, E. P. J. 1999, ApJ, 513, L45

Long, M., Romanova, M. M., \& Lovelace, R. V. E. 2008, MNRAS, 386, 1274

Pons, J. A. \& Geppert, U. 2007, A\& A, 470, 303 